\title{
BL Lac identification for the ultraluminous X-ray source observed in the direction of NGC 4698
}

\author{
L. Foschini ${ }^{1}$, L. C. $\mathrm{Ho}^{2}$, N. Masetti ${ }^{1}$, M. Cappi ${ }^{1}$, M. Dadina ${ }^{1}$, L. Bassani ${ }^{1}$, G. Malaguti ${ }^{1}$, E. Palazzi ${ }^{1}$, \\ G. Di Cocco ${ }^{1}$, P. Martini ${ }^{2}$, S. Ravindranath ${ }^{2,3}$, J. B. Stephen ${ }^{1}$, M. Trifoglio ${ }^{1}$, and F. Gianotti ${ }^{1}$ \\ ${ }^{1}$ Istituto di Astrofisica Spaziale e Fisica Cosmica (IASF-CNR) - Sezione di Bologna ${ }^{\star}$, via Gobetti 101, 40129, Bologna, Italy \\ 2 The Observatories of the Carnegie Institution of Washington, 813 Santa Barbara Street, Pasadena, CA 91101, USA \\ ${ }^{3}$ Department of Astronomy, University of California, Berkeley, CA 94720, USA
}

Received 5 August 2002 / Accepted 20 September 2002

\begin{abstract}
We report the identification of the optical and radio counterparts of the ultraluminous X-ray (ULX) source XMMU J124825.9+083020 (NGC 4698-ULX1). The optical spectrum taken with the VLT yields a redshift of $z=0.43$, which implies that the ULX is not associated with the nearby galaxy NGC 4698. The spectral energy distribution calculated from the available data indicates that the source is likely to be a BL Lac object. The possible synchrotron peak at X-ray energies suggests that this source may be a $\gamma$-ray emitter.
\end{abstract}

Key words. galaxies: active - galaxies: Seyfert - galaxies: BL Lacertae objects: general

\section{Introduction}

In recent years, the improved imaging capabilities and increased sensitivity of ROSAT, Chandra and XMM-Newton have allowed us to effectively study discrete sources in nearby galaxies beyond the Local Group. Particularly intriguing is the discovery of off-nuclear X-ray sources with luminosities well above the Eddington limit for a typical neutron star, $\sim 10^{38} \mathrm{erg} \mathrm{s}^{-1}$, and up to $2 \times 10^{40} \mathrm{erg} \mathrm{s}^{-1}$ (e.g., Read et al. 1997; Colbert \& Mushotzky 1999; Roberts \& Warwick 2000; Makishima et al. 2000; Fabbiano et al. 2001). These sources are typically called ultraluminous X-ray sources (ULXs). Despite much effort, little is presently known about these sources. The identification of their optical counterparts is often problematical when the sources are superposed against regions of high surface brightness in the host galaxy. To date, only two ULXs appear to have a clear optical identification (Roberts et al. 2001; Wu et al. 2002), while for others it has been possible to study only the nearby environment (Pakull \& Mirioni 2002; Wang 2002).

We have started a search for ULXs in a sample of nearby galaxies (Foschini et al. 2002). This paper concerns followup observations of the ULX in NGC 4698. We discuss the radio and optical counterparts and give a redshift estimate.

\footnotetext{
Send offprint requests to: L. Foschini, e-mail: foschini@bo.iasf.cnr.it

^ Formerly Istituto TeSRE - CNR.
}

We identify the source with a background source, most likely a BL Lac object at $z=0.43$.

\section{X-ray data (XMM-Newton)}

NGC 4698 is an Sab spiral galaxy located in the Virgo cluster $(d=16.8 \mathrm{Mpc})$. It hosts an active nucleus, classified as a Seyfert 1.9 by Ho et al. (1997). The galaxy was observed on 16 December 2001 using the European Photon Imaging Camera (EPIC) on board the XMM-Newton satellite. EPIC is composed of two instruments: the PN-CCD camera (Strüder et al. 2001) and two MOS-CCD detectors (Turner et al. 2001). The effective exposure time was $9.2 \mathrm{ks}$.

We find only one ULX apparently associated with NGC 4698 (Foschini et al. 2002), located 73" from the optical nucleus of the galaxy, at $\alpha=12^{\mathrm{h}} 48^{\mathrm{m}} 25.9^{\mathrm{s}}$ and $\delta=+08^{\circ} 30^{\prime} 20^{\prime \prime}$ (J2000). The point source centroid is measured to precision of better than $0 ! ' 1$, and the absolute pointing uncertainty is $<4^{\prime \prime}$ (Jansen et al. 2001). We detected 198 photons with MOS1, in a circle of radius $30^{\prime \prime}, 249$ with MOS2, and 414 with PN. These statistics are sufficient to perform spectral fitting with simple models. The ones we use are the power law (PL), black body (BB), bremsstrahlung (BR), and the multicolor black body disk (MCD, diskbb in Xspec) by Mitsuda et al. (1984). Fluxes were corrected according to the energy encircled fraction (Ghizzardi 2001).

For the processing, screening, and analysis of the data we used the standard tools in the XMM-SAS software (v. 5.2) and 


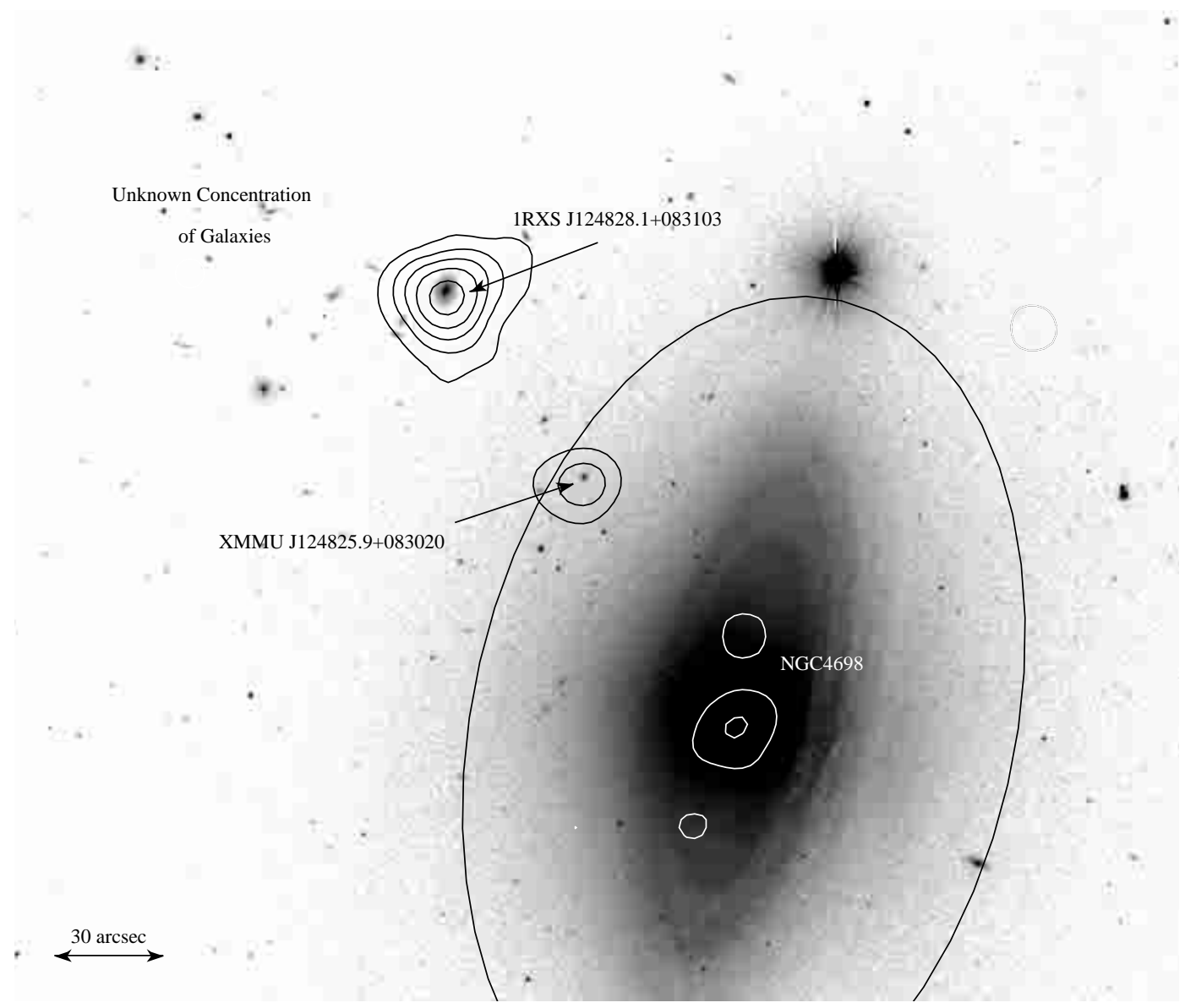

Fig. 1. $R$-band image from VLT-U3/FORS1, superimposed in contours the smoothed image from the XMM-Newton EPIC-MOS2 data in the 0.5-10 keV energy band (white over NGC 4698, black in the remaining field). North is up and East to the left. The $D_{25}$ ellipse (3'8) is shown for comparison.

HEAsoft Xspec (v. 11.0.1). Correction for vignetting has not been applied because the source is close to the center of the field of view $\left(<2^{\prime}\right)$ and most of the detected photons have energies less than $5 \mathrm{keV}$ (see Lumb 2002).

The best fit (Fig. 3) is found using the power-law model with $\Gamma=2.0 \pm 0.2\left(\chi^{2}=18.3, v=24\right)$, giving a flux of $8.6 \times$ $10^{-14} \mathrm{erg} \mathrm{cm}^{-2} \mathrm{~s}^{-1}$. If the source is located in NGC 4698, for which we assume a distance of $16.8 \mathrm{Mpc}$, the corresponding luminosity, assuming isotropic emission, is $\sim 3 \times 10^{39} \mathrm{erg} \mathrm{s}^{-1}$. For $z=0.43$, as discussed below, the luminosity becomes $3.9 \times$ $10^{43} \mathrm{erg} \mathrm{s}^{-1}$. Additional information on the fits using the other models is given in Table 1 .

\section{Radio data (VLA)}

The VLA observations of NGC 4698 were performed by Ho \& Ulvestad (2001) on 29 August $1999(20 \mathrm{~cm})$ and 31 October $1999(6 \mathrm{~cm})$. The source detection at $6 \mathrm{~cm}$ was reported by Ho \& Ulvestad (2001); the flux density is $1.13 \mathrm{mJy}$ and the background noise is $0.072 \mathrm{mJy}_{\text {beam }}{ }^{-1}$. The source, which appears largely unresolved at a resolution of $\sim 1^{\prime \prime}$, is located at $\alpha=12^{\mathrm{h}} 48^{\mathrm{m}} 25.9^{\mathrm{s}}$ and $\delta=+08^{\circ} 30^{\prime} 21^{\prime \prime}(\mathrm{J} 2000)$; the radio position is accurate to $\sim 0 . ' 1$.

A reanalysis of the VLA data detected the source also at $20 \mathrm{~cm}$. It is unresolved with the $1^{\prime \prime}$ beam, and its

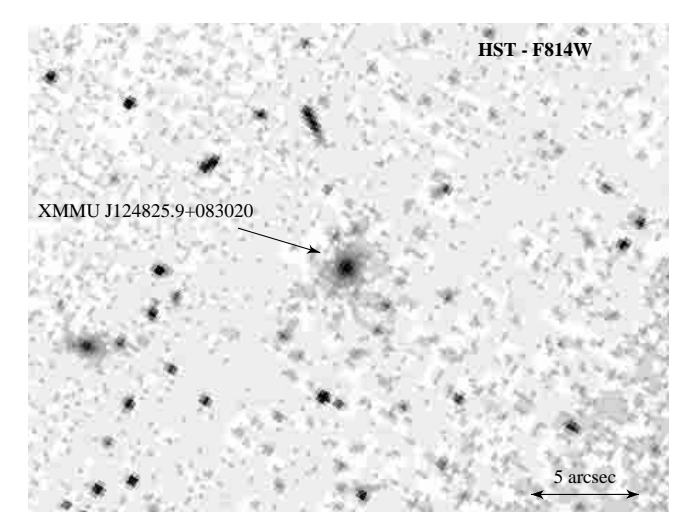

Fig. 2. Archival Hubble Space Telescope image of the region around XMMU J124825.9 + 083020 taken in the F814W filter. North is up and East to the left.

$20 \mathrm{~cm}$ position is consistent with that measured at $6 \mathrm{~cm}$. We find a flux density of $0.34 \mathrm{mJy}$, with a background noise of $0.032 \mathrm{mJy} \mathrm{beam}^{-1}$. The spectral index, defined as $S_{v} \propto v^{\alpha}$, is $\alpha_{\mathrm{r}} \approx 1.0$. 
Table 1. Results from the fit of the X-ray data. Columns: (1) Model: power law (PL), black body (BB), bremsstrahlung (BR), multicolor black body disk (MCD); (2) free parameter of the model: photon index $\Gamma$ for PL, temperature $(\mathrm{keV})$ for BB and BR, temperature $(\mathrm{keV})$ at the inner disk for MCD; (3) $\chi^{2}$ and degrees of freedom of the spectral fitting; (4) flux in the $0.5-10 \mathrm{keV}$ band $\left(10^{-14} \mathrm{erg} \mathrm{cm}^{-2} \mathrm{~s}^{-1}\right)$; (5) X-ray luminosity in the $0.5-10 \mathrm{keV}$ band $\left(10^{43} \mathrm{erg} \mathrm{s}^{-1}\right)$ calculated for $z=0.43, H_{0}=75 \mathrm{~km} \mathrm{~s}^{-1} \mathrm{Mpc}^{-1}$ and $q_{0}=0.5$. No absorption is required in excess of the the Galactic column, $N_{\mathrm{H}}=1.87 \times 10^{20} \mathrm{~cm}^{-2}$. The uncertainties in the parameters are at the $90 \%$ confidence level.

\begin{tabular}{lcccc}
\hline \hline $\begin{array}{l}\text { Model } \\
(1)\end{array}$ & Parameter & $\begin{array}{c}\chi^{2} / \text { d.o.f. } \\
(2)\end{array}$ & $F_{X}$ & $L_{X}$ \\
\hline $\mathrm{PL}$ & $\Gamma=2.0 \pm 0.2$ & $18.3 / 24$ & 8.6 & 3.9 \\
$\mathrm{BB}$ & $k T=0.35 \pm 0.05$ & $34.5 / 24$ & 4.5 & \\
$\mathrm{BR}$ & $k T=2 \pm 1$ & $19.0 / 24$ & 6.7 & \\
$\mathrm{MCD}$ & $k T_{i n}=0.6 \pm 0.1$ & $23.7 / 24$ & 5.4 & \\
\hline
\end{tabular}

\section{Optical data}

\subsection{Digitized Sky Survey (DSS)}

The optical counterpart of the source is visible on the DSS and the HST observations of NGC 4698. We are certain of the identification from the relative offset between the nucleus and the optical counterpart. From the Automatic Plate Measurement $\left(\mathrm{APM}^{1}\right)$ catalog, the point source has $R=18.9 \mathrm{mag}$ and $B=$ $21.9 \mathrm{mag}$, indicating a very red color $(B-R=3.0 \mathrm{mag})$. The original observation was made with the 48 inch Schmidt telescope at Palomar Observatory on 17 February 1950.

\subsection{Hubble Space Telescope (HST)}

We retrieved the calibrated F450W, F606W, and F814W WFPC2 images of NGC 4698 from the HST Archive $^{2}$ to perform photometry of the optical counterpart of the source. The observations with the F450W and F814W filters were performed on 10 August 2001, while the observation with F606W was taken on 31 March 2000. In all the filters, the source is located on the WF detector, which has a scale of $0 . ' 1$ per pixel. The total exposure time is $460 \mathrm{~s}(2 \times 230 \mathrm{~s})$ in the F450W and F814W filters and $600 \mathrm{~s}$ (400 s and $200 \mathrm{~s})$ in the F606W filter. We combined the separate images taken in each filter using the crrej task in IRAF to reject cosmic rays. Aperture photometry was done using the tasks available in the apphot package. The integrated magnitudes, determined using the aperture growth curve method, were transformed to the Vega magnitude system using the zeropoints given in the HST Data Handbook. We find $m_{\mathrm{F} 450 \mathrm{~W}}=21.1 \pm 0.1 \mathrm{mag}$, $m_{\mathrm{F} 606 \mathrm{~W}}=19.9 \pm 0.1 \mathrm{mag}$, and $m_{\mathrm{F} 814 \mathrm{~W}}=18.6 \pm 0.1 \mathrm{mag}$.

\footnotetext{
1 http://www . ast.cam.ac.uk/ apmcat/

2 Based on observations made with the NASA/ESA Hubble Space Telescope, obtained from the data archive at the Space Telescope Institute. STScI is operated by the association of Universities for Research in Astronomy, Inc. under the NASA contract NAS 5-26555.
}

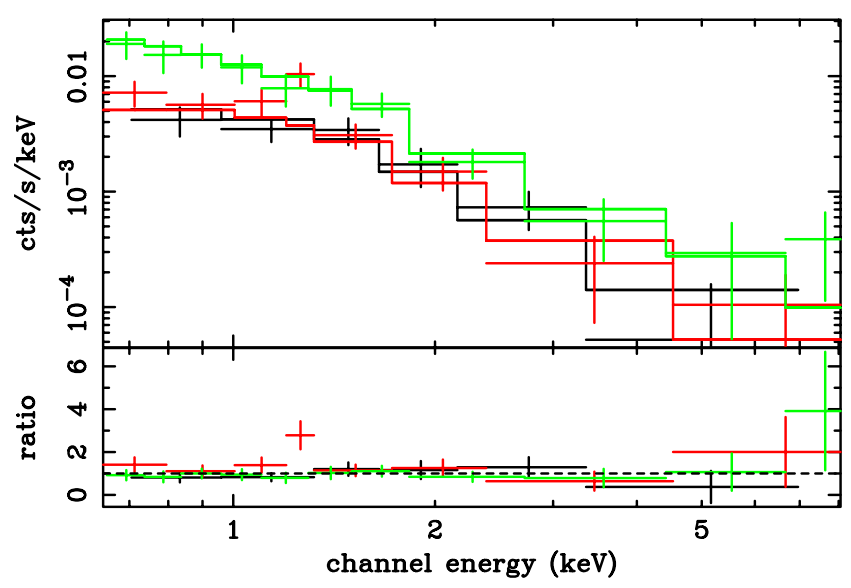

Fig. 3. XMM-Newton spectrum of XMMU J124825.9+083020. Data are from MOS1, MOS2, and PN.

In the Johnson-Kron-Cousins system these values translate into $B=21.3 \pm 0.1 \mathrm{mag}, R=19.6 \pm 0.1 \mathrm{mag}$, and $I=18.5 \pm 0.1 \mathrm{mag}$.

\subsection{Las Campanas (Magellan)}

XMMU J124825.9+083020 was observed for $900 \mathrm{~s}(3 \times$ 300 s) on 6 April 2002 with the LDSS-2 spectrograph (Allington-Smith et al. 1994) on the $6.5 \mathrm{~m}$ Baade telescope at Las Campanas Observatory. We observed through a 1'. 25 longslit in 0.' 9 seeing, with the slit oriented at the parallactic angle. We used the medium red grism, which gives a dispersion of $5.3 \AA$ pixel $^{-1}$. The $F W H M$ spectral resolution was $16 \AA$, and the spatial resolution was 0 .'38 pixel $^{-1}$.

Basic data reduction was performed using the IRAF package. The individual spectroscopic frames were corrected for overscan, flat-fielded using domeflats and summed to obtain the final object frame. The spectral extraction was done by summing the counts within an aperture of 6 pixels $\left(2{ }^{\prime \prime} 3\right)$. Wavelength calibration was achieved using a polynomial fit to the lines in the sky spectrum.

The spectrum in the range 5000-7000 $\AA$ is approximately described by the form $f_{\lambda} \approx \lambda^{4}$.

\subsection{Very Large Telescope (VLT)}

VLT observations ${ }^{3}$ were performed with VLT-UT3 (Melipal) plus the FORS1 spectrograph. FORS1 is equipped with a $2048 \times 2048$ pixel Tektronix CCD, which covers a 6.8 $\times$ 6.8 field in the standard resolution imaging mode with a scale of 0.2 pixel $^{-1}$.

Two spectra, both with an exposure time of $570 \mathrm{~s}$, were acquired on 21 April 2002, starting at 4:52:50 UT. The spectra were acquired using Grism \#150I plus order separator GG435, which avoids overlapping of spectral orders over a given wavelength; this limited the spectral range to 4500-9000 ̊. The slit width was $1^{\prime \prime}$ for both spectra, and this setup secured a

\footnotetext{
3 Based on observations made with ESO Telescopes at the Paranal Observatory under programme ID 269.D - 5014 .
} 


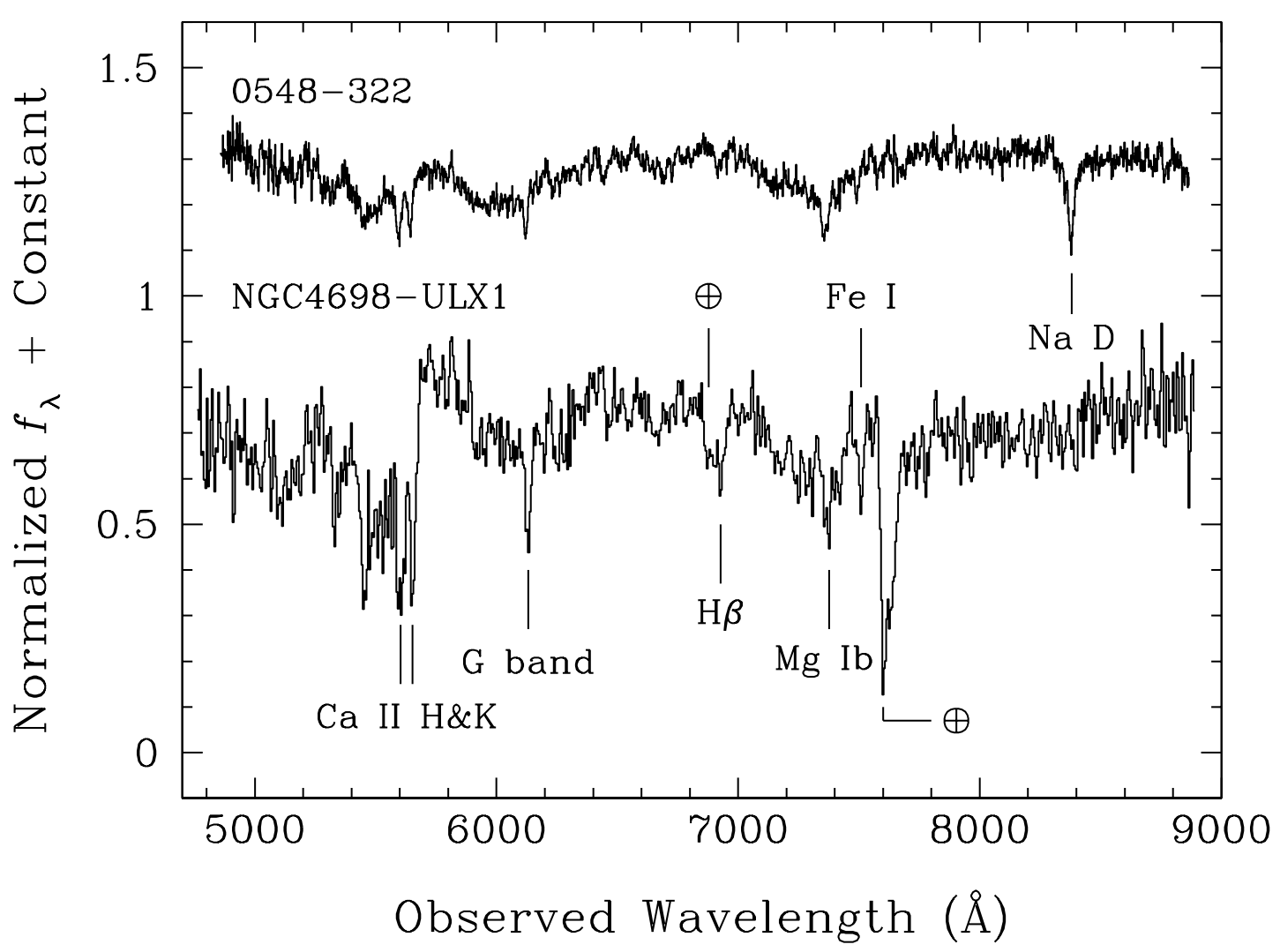

Fig. 4. Normalized VLT spectrum with identifications of absorption lines marked. Telluric absorption lines are marked with the symbol $\oplus$. Shown for comparison is the spectrum of the BL Lac object 0548-322 (Barth et al. 2002), redshifted to $z=0.43$.

final dispersion of $5.5 \AA$ pixel $^{-1}$, corresponding to a $F W H M$ resolution of $13 \AA$. Before the spectroscopic observation, a one minute $R$-band acquisition image was obtained on the same night starting at 04:42:52 UT under very good seeing conditions $\left.(\sim 0)^{\prime} 6\right)$. The object appeared clearly elliptical and extended, with a bright core and a fuzzy halo. Moreover, an irregular spot located around 1" north of the object core is apparent in the VLT image.

After correction for flat-field and bias, the spectra were background subtracted and optimally extracted (Horne 1986) using IRAF. He-Ne-Ar and $\mathrm{Hg}-\mathrm{Cd}$ lamps were used for wavelength calibration. The wavelength calibration was checked against the position of night sky lines; the typical error was $0.5 \AA$. Finally, the two spectra were stacked together in order to increase the signal-to-noise ratio. We encountered problems during observation of the spectrophotometric standard, and hence our spectra are not flux calibrated.

Figure 4 shows the normalized VLT spectrum, with several absorption lines identified (see Laurent-Muehleisen et al. (1998) for a discussion on optical identification of BL Lacs). The detected lines include Ca II H\&K $\lambda \lambda 3933,3968$, the $G$ band $\lambda 4304, \mathrm{H} \beta \lambda 4861$, and Fe I $\lambda 5270$ : they all indicate $z \approx 0.43$. The line strengths are heavily diluted by the featureless continuum, but the spectrum shares close similarity to that of the BL Lac object 0548-322 (Barth et al. 2002). We shifted the spectrum of 0548-322, which has a redshift of 0.069 , to $z=0.43$.

\section{Discussion}

\subsection{Classification}

The absorption features detected in the VLT spectrum clearly place XMMU J124825.9+083020 at $z=0.43$. We identify the source as a BL Lac object, for the following reasons. (1) We detect no emission lines; the upper limit to any emission feature is $\sim 5 \AA$. (2) The stellar features superposed on the featureless continuum have strengths generally consistent with those of other BL Lac objects. We demonstrate this concretely by comparing a redshifted spectrum of the BL Lac object 0548-322 (Fig. 4). The break contrast at $4000 \AA$ is $\sim 29 \%$, again similar to other BL Lac objects (Laurent-Muehleisen et al. 1998). (3) The optical continuum slope measured in the Magellan spectrum is consistent with those of high-frequency peaked BL Lac objects (e.g., Stickel et al. 1993). And (4) the multiwavelength spectral energy distribution (Fig. 5), although not assembled from simultaneous observations, is highly reminiscent of those of BL Lac objects.

By using two-point spectral indices, namely the radio-tooptical $\alpha_{\text {ro }}$ and optical-to-X-ray $\alpha_{\text {ox }}$, it is possible to show that different objects populate different regions of the $\alpha_{\mathrm{ro}}-$ $\alpha_{\text {ox }}$ plane (e.g., Brinkmann et al. 1997; Laurent-Muehleisen et al. 1999). Another method has been suggested by Maccacaro et al. (1988), who proposed a nomograph to link the X-ray flux in the energy band $0.3-3.5 \mathrm{keV}$ and the visual magnitude. The values of $\alpha_{\text {ro }}$ and $\alpha_{\mathrm{ox}}$ for the present source are 0.42 
and 0.95 , respectively, thus placing it in the region of X-ray selected BL Lacs (Brinkmann et al. 1997), or high-energy peaked BL Lacs in the $\alpha_{\text {ro }}-\alpha_{\text {ox }}$ diagram of Laurent-Muehleisen et al. (1999). The nomograph of Maccacaro et al. (1988) gives a ratio $f_{\mathrm{x}} / f_{\mathrm{v}}$ between 1.3 and 3.8 (depending on whether we use the HST optical magnitudes through the F606W or F450W filter, respectively), in the regime of AGNs and BL Lacs.

The spectral indices can be used to deduce some general properties of the dominant radiation mechanism. If $\alpha_{\mathrm{x}} \leq \alpha_{\mathrm{r}}$, the source may exhibit relativistic beaming, while if $\alpha_{\mathrm{x}}>\alpha_{\mathrm{r}}$, it may fulfill the conditions of the homogeneous synchrotron model (Harris \& Krawczynski 2002). In our case, we have $\alpha_{\mathrm{x}} \approx$ $\alpha_{\mathrm{r}} \approx 1$, so that we cannot clearly discriminate between these two cases. If the source is a high-frequency peaked BL Lac, however, it is likely that the homogeneous synchrotron model is more applicable. This is confirmed by the $\alpha_{\text {xox }}$ test of Sambruna et al. (1996): in this case, the difference by $\alpha_{\mathrm{ox}}-\alpha_{\mathrm{x}}=\alpha_{\mathrm{xox}}$ is approximately equal to zero, so avoiding a clear discrimination between the physical mechanism of the source.

The spectral energy distribution (Fig. 5) is comparable to those of BL Lac objects peaked in the $\gamma$-ray domain (e.g., Fossati et al. 1998), but the third EGRET catalog (Hartman et al. 1999) does not have any source within several degrees of XMMU J124825.9+083020.

\subsection{Search for ULXs and Contamination with Background Objects}

This research is a useful demonstration of just how difficult it is to identify the physical nature of ULXs. It shows the vital importance of redshift determinations. Although we had a lot of photometric data on XMMU J124825.9 + 083020, by themselves they were insufficient to clearly establish whether the source belongs to NGC 4698 or is a background object. In the first case, the source would have been something similar to a microquasar (e.g., Mirabel \& Rodríguez 1999), probably located in a globular cluster. Indeed, the optical image of the source appeared to be slightly extended (angular extent $\sim 3^{\prime \prime}$ ), and its position with respect to NGC 4698 suggested that it could be a globular cluster, albeit an unusually large one. The possibility of an accreting black hole in a globular cluster is not so remote: Chandra observations of the globular cluster system of NGC 4472 show that about $40 \%$ of the bright low mass $\mathrm{X}$-ray binaries are associated with optically identified globular clusters (Kundu et al. 2002). In addition, the X-ray luminosity function shows a break near $3 \times 10^{38} \mathrm{erg} \mathrm{s}^{-1}$, suggesting that the brightest X-ray binaries are accreting black holes (Kundu et al. 2002), perhaps microquasars.

XMM-Newton observations of the Lockman Hole (Hasinger et al. 2001) show that the number of background sources in the energy band $0.5-2 \mathrm{keV}$ with flux greater than $4.0 \times 10^{-14} \mathrm{erg} \mathrm{cm}^{-2} \mathrm{~s}^{-1}$ (the best fit value from Table 1) is $15 \mathrm{deg}^{-2}$. In the energy band $2-10 \mathrm{keV}$, there are 40 sources deg ${ }^{-2}$ with flux higher than $4.6 \times 10^{-14} \mathrm{erg} \mathrm{cm}^{-2} \mathrm{~s}^{-1}$. Assuming the same $\log N-\log S$ relation, and considering that the $D_{25}$ area of NGC 4698 is about $7.9 \operatorname{arcmin}^{2}$, we expect 0.08 background objects in the $2-10 \mathrm{keV}$ energy band

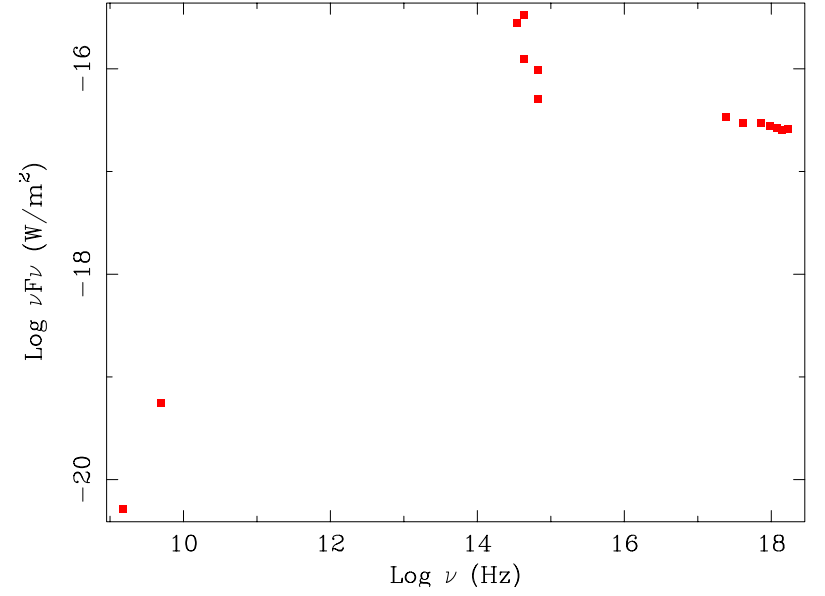

Fig. 5. The spectral energy distribution of XMMU J124825.9+083020, assembled from data taken with the VLA, DSS, HST, and XMM.

and 0.03 in the energy band $0.5-2 \mathrm{keV}$. However, despite these low values, we have found, in the present case, that the only ULX is a background AGN.

The above calculations could be underestimated in the present case because the VLT images show an unknown concentration of galaxies north-east of NGC 4698 (see Fig. 1), thus suggesting the possibility of a statistically meaningful excess of background sources. However, no additional X-ray sources is seen in the present XMM-Newton observation. Perhaps, a longer exposure may reveal soft X-ray emission or additional sources, if NGC 4698 lies along the line of sight to a galaxy cluster. The three X-ray sources identified to date, however, have three redshifts: XMMU J124825.9+083020 has $z=0.43$, NGC 4698 has $z=0.0033$, and the ROSAT source 1RXS J124828.1+083103 has been recently identified with a Seyfert nucleus at $z=0.12$ (Xu et al. 2001). Therefore, these three sources are not members of a single cluster.

\section{Final remarks}

We have presented a multiwavelength analysis of the ultraluminous X-ray source XMMU J124825.9+083020 observed in the direction of NGC 4698. The availability of X-ray, optical, and radio data makes it possible to construct the spectral energy distribution, but the crucial information to decipher the true nature of the source was provided by the optical spectrum. We show that the absorption features are unambiguously associated with a background source at $z=0.43$. We argue that the source is most likely a BL Lac object, most likely of the highfrequency peaked category, based on the absence of emission lines, the dilution of the absorption features, the slope of the optical continuum, and the overall shape of the spectral energy distribution.

Acknowledgements. We would like to thank A. Pizzella for useful discussion about NGC 4698, the Service Mode Observations personnel at ESO, and in particular M. Romaniello, for the help in the preparation of VLT observations. This work is based on observations obtained with XMM-Newton, an ESA science mission with instruments and contributions directly funded by ESA Member States and the 
USA (NASA). This research has made use of the NASA Astrophysics Data System Abstract Service and of the NASA/IPAC Extragalactic Database (NED), which is operated by the Jet Propulsion Laboratory, California Institute of Technology, under contract with the National Aeronautics and Space Administration. We acknowledge the partial support of the Italian Space Agency (ASI) to this research. L.C.H. is grateful for financial support from the Carnegie Institution of Washington and from NASA grants.

\section{References}

Allington-Smith, J., Breare, M., Ellis, R., et al. 1994, PASP, 106, 983 Barth, A. J., Ho, L. C., \& Sargent, W. L. W. 2002, ApJ, in press

Brinkmann, W., Siebert, J., Feigelson, E. D., et al. 1997, A\&A, 323, 739

Colbert, E. J. M., \& Mushotzky, R. F. 1999, ApJ, 519, 89

Fabbiano, G., Zezas, A., \& Murray, S. S. 2001, ApJ, 554, 1035

Foschini, L., Di Cocco, G., Ho, L. C., et al. 2002, A\&A, 392, 817

Fossati, G., Maraschi, L., Celotti, A., et al. 1998, MNRAS, 299, 433

Ghizzardi, S. 2001, in flight calibration of the PSF for the MOS1 and MOS2 cameras. EPIC-MCT-TN-011 (Internal report)

Jansen, F., Lumb, D., Altieri, B., et al. 2001, A\&A, 365, L1

Hamuy, M., Walker, A. R., Suntzeff, N. B., et al. 1992, PASP, 104, 533

Hamuy, M., Suntzeff, N. B., Heathcote, S. R., et al. 1994, PASP, 106, 566

Harris, D. E., \& Krawczynski, H. 2002, ApJ, 565, 244

Hartman, R. C., Bertsch, D. L., Bloom, S. D., et al. 1999, ApJS, 123, 79

Hasinger, G., Altieri, B., Arnaud, M., et al. 2001, A\&A, 365, L45

Ho, L. C., Filippenko, A. V., \& Sargent, W. L. W. 1997, ApJS, 112, 315
Ho, L. C., \& Ulvestad, J. S. 2001, ApJS, 133, 77

Horne, K. 1986, PASP, 98, 609

King, A. R., Davies, M. B., Ward, M. J., et al. 2001, ApJ, 552, L109

Kundu, A., Maccarone, T. J., \& Zepf, S. E. 2002, ApJ, 574, L5

Laurent-Muehleisen, S. A., Kollgaard, R. I., Ciardullo, R., et al. 1998, ApJS, 118, 127

Laurent-Muehleisen, S. A., Kollgaard, R. I., Feigelson, E. D., et al. 1999, ApJ, 525, 127

Lumb, D. H. 2002, Proc. Symp. New Visions of the X-ray Universe in the XMM-Newton and Chandra Era, ed. F. Jansen, ESA SP488 [astro-ph/0203278]

Maccacaro, T., Gioia, I. M., Wolter, A., et al. 1988, ApJ, 326, 680

Makishima, K., Kubota, A., Mizuno, T., et al. 2000, ApJ, 535, 632

Mirabel, I. F., \& Rodríguez, L. F. 1999, ARA\&A, 37, 409

Mitsuda, K., Inoue, H., Koyama, K., et al. 1984, PASJ, 36, 741

Pakull, M. W., \& Mirioni, L. 2002, Proc. Symp. New Visions of the $\mathrm{X}$-ray Universe in the XMM-Newton and Chandra Era, ed. F. Jansen, ESA SP488 [astro-ph/0202488]

Read, A. M., Ponman, T. J., \& Strickland, D. K. 1997, MNRAS, 286, 626

Roberts, T. P., \& Warwick, R. S. 2000, MNRAS, 315, 98

Roberts, T. P., Goad, M. R., Ward, M. J., et al. 2001, MNRAS, 325, L7

Sambruna, R., Maraschi, L., \& Urry, C. M. 1996, ApJ, 463, 444

Stickel, M., Fried, J. W., \& Kühr, H. 1993, A\&AS, 98, 393

Strüder, L., Briel, U., Dennerl, K., et al. 2001, A\&A, 365, L18

Turner, M. J., Abbey, A., Arnaud, M., et al. 2001, A\&A, 365, L27

Xu, D. W., Wei, J. Y., \& Hu, J. Y. 2001, Chinese J. Astron. Astrophys., 1,46

Wang, Q. D. 2002, MNRAS, 332, 764

Wu, H., Xue, S. J., Xia, X. Y., et al. 2002, ApJ, 577, 738 